Vol. 39 (1989) [287-299]

\title{
GENERALISED CONVEXITY AND DUALITY IN MULTIPLE OBJECTIVE PROGRAMMING
}

\author{
T. Weir AND B. MOND
}

\begin{abstract}
By considering the concept of weak minima, different scalar duality results are extended to multiple objective programming problems. A number of weak, strong and converse duality theorems are given under a variety of generalised convexity conditions.
\end{abstract}

\section{INTRODUCTION}

Consider the multiple objective optimisation problem:

(P) minimise $f(x)$ subject to $g(x) \leqq 0$

where $f: \mathbf{R}^{n} \rightarrow \mathbf{R}^{k}$ and $g: \mathbf{R}^{n} \rightarrow \mathbf{R}^{m}$. Several approaches to duality for the multiple objective optimisation problem may be found in the literature. These include the use of vector valued Lagrangians $[13,16,17]$ and Lagrangians incorporating matrix Lagrange multipliers $[3,4,7$ and 9$]$.

In Weir [16], using a vector-valued Lagrangian, the well-known duality results of Wolfe [18] for scalar valued convex programs were extended to the multiple objective optimisation problem. The approach there was to consider the properly efficient solutions [8] of $(P)$ and to relate primal and dual properly efficient solutions. Some restricted results on duality for non-convex multiple objective optimisation problems were also given.

In this paper, by considering the concept of a weak minimum, a complete generalisation of the scalar duality results of Wolfe [18] and those of Mond and Weir [12] and Bector and Bector [2] will be described for the multiple objective optimisation problem. The results also include generalisations of the converse duality results of Mond and Weir [12] and Bector and Bector [2] and the strict converse duality results of Weir [14] , [15] and Bector and Bector [2].

\section{Preliminaries}

Throughout this paper the following conventions for vectors in $\mathbf{R}^{\mathbf{n}}$ will be followed:

Received 16 May 1988

Copyright Clearance Centre, Inc. Serial-fee code: 0004-9729/89 \$A2.00+0.00. 


$$
\begin{aligned}
& x>y \text { if and only if } x_{i}>y_{i}, i=1,2, \ldots, n, \\
& x \geqq y \text { if and only if } x_{i} \geqq y_{i}, i=1,2, \ldots, n, \\
& x \geqslant y \text { if and only if } x_{i} \geqq y_{i}, i=1,2, \ldots, n \text { but } x \neq y, \\
& x \ngtr y \text { is the negation of } x>y .
\end{aligned}
$$

For the problem $(P)$, a point $x_{0}$ is said to be a weak minimum if there exists no other feasible point $x$ for which $f\left(x_{0}\right)>f(x)$.

The following Fritz John and Kuhn-Tucker theorems [6] will be needed:

Theorem 2.1. Let $(P)$ have a weak minimum at $x=x_{0}$. Then there exists $\lambda \in \mathbf{R}^{k}, y \in \mathbf{R}^{m}$ such that

$$
\begin{aligned}
\nabla \lambda^{t} f\left(x_{0}\right)+\nabla y^{t} g\left(x_{0}\right) & =0 \\
y^{t} g\left(x_{0}\right) & =0, \\
(\lambda, y) & \geqslant 0 .
\end{aligned}
$$

Theorem 2.2. Let $x_{0}$ be a weak minimum for $(P)$ at which the Kuhn-Tucker constraint qualification is satisfied. Then there exists $\lambda \in \mathbf{R}^{k}, y \in \mathbf{R}^{m}$ such that

$$
\begin{aligned}
\nabla \lambda^{t} f\left(x_{0}\right)+\nabla y^{t} g\left(x_{0}\right) & =0, \\
y^{t} g\left(x_{0}\right) & =0, \\
y & \geqq 0, \\
\lambda \geqslant 0, \lambda^{t} e & =1,
\end{aligned}
$$

where $e=(1,1, \ldots, 1) \in \mathrm{R}^{k}$.

\section{DUAlity}

In relation to $(P)$ consider the problem

(D) maximise $f(u)+y^{t} g(u) e$

$$
\text { subject to } \begin{aligned}
\nabla \lambda^{t} f(u)+\nabla y^{t} g(u) & =0, \\
y & \geqq 0, \\
\lambda & \in \Lambda,
\end{aligned}
$$

where $\Lambda=\left\{\lambda \in \mathbf{R}^{k}: \lambda \geqslant 0, \lambda^{t} e=1\right\}$. 
The problem $(D)$ may be regarded as a multiple objective Wolfe [18] dual for $(P)$.

Theorem 3.1. (Weak Duality). If, for all feasible $(x, u, y, \lambda)$,

(a) $f+y^{t}$ ge is pseudoconvex; or

(b) $\lambda^{t} f+y^{t} g$ is pseudoconvex, then $f(x) \nless f(u)+y^{t} g(u) e$.

\section{ProOF:}

(a) Let $x$ be feasible for $(P)$ and $(u, y, \lambda)$ feasible for $(D)$. Suppose $f_{i}(x)<f_{i}(u)+$ $y^{t} g(u)$ for all $i=1,2, \ldots, k$. Then $f_{i}(x)+y^{t} g(x)<f_{i}(u)+y^{t} g(u)$ for all $i=1,2, \ldots, k$. The pseudoconvexity of $f+y^{t} g e$ implies that

$$
(x-u)^{t} \nabla\left\{f_{i}(u)+y^{t} g(u)\right\}<0
$$

and hence

$$
(x-u)^{t}\left\{\nabla \lambda^{t} f(u)+\nabla y^{t} g(u)\right\}<0
$$

which contradicts the constraint $(8)$ of $(D)$.

(b) Let $x$ be feasible for $(P)$ and $(u, y, \lambda)$ feasible for $(D)$. Suppose $f_{i}(x)<f_{i}(u)+$ $y^{t} g(u)$ for all $i=1,2, \ldots, k$. Then $f_{i}(x)+y^{t} g(x)<f_{i}(u)+y^{t} g(u)$ for all $i=1,2, \ldots, k$. Thus, $\lambda^{t} f(x)+y^{t} g(x)<\lambda^{t} f(u)+y^{t} g(u)$ and the pseudoconvexity of $\lambda^{t} f+y^{t} g$ implies that

$$
(x-u)^{t}\left\{\nabla \lambda^{t} f(u)+\nabla y^{t} g(u)\right\}<0
$$

which contradicts the constraint $(8)$ of $(D)$.

Theorem 3.2. (Strong Duality). Let $x_{0}$ be a weak minimum for $(P)$ at which the Kuhn-Tucker constraint qualification is satisfied. Then there exist $(y, \lambda)$ such that $\left(x_{0}, y, \lambda\right)$ is feasible for $(D)$ and the objective values of $(P)$ and $(D)$ are equal. If, also, (a) $f+y^{t} g e$ is pseudoconvex or (b) $\lambda^{t} f+y^{t} g$ is pseudoconvex then $\left(x_{0}, y, \lambda\right)$ is a weak maximum for $(D)$.

Proof: Since $x_{0}$ is a weak minimum for $(P)$ at which the Kuhn-Tucker constraint qualification is satisfied, then by Theorem 2.2 , there exist $y \geqq 0, \lambda \geqslant 0, \lambda^{t} e=1$ such that $\nabla \lambda^{t} f\left(x_{0}\right)+\nabla y^{t} g\left(x_{0}\right)=0, y^{t} g\left(x_{0}\right)=0$.

Thus $\left(x_{0}, y, \lambda\right)$ is feasible for $(D)$ and clearly the objective values of (P) and (D) are equal.

If $\left(x_{0}, y, \lambda\right)$ is not a weak maximum for $(D)$ then there exists feasible $\left(u^{*}, y^{*}, \lambda^{*}\right)$ for $(D)$ such that

$$
f_{i}\left(u^{*}\right)+y^{* t} g\left(u^{*}\right)>f_{i}\left(x_{0}\right)+y^{* t} g\left(x_{0}\right) \text { for all } i=1,2, \ldots, k .
$$

(a) Since $f+y^{t} g e$ is pseudoconvex, $\left(x_{0}-u\right)^{t} \nabla\left(f_{i}\left(u^{*}\right)+y^{* t} g\left(u^{*}\right)\right)<0$ for all $i=$ $1,2, \ldots, k$. Thus $\left(x_{0}-u^{*}\right)^{t}\left\{\nabla \lambda^{* t} f\left(u^{*}\right)+\nabla y^{* t} g\left(u^{*}\right)\right\}<0$ contradicting the feasibility of $\left(u^{*}, y^{*}, \lambda^{*}\right)$. 
Thus $\left(x_{0}, y, \lambda\right)$ is a weak maximum for $(D)$.

(b) Since $f_{i}\left(u^{*}\right)+y^{* t} g\left(u^{*}\right)>f_{i}\left(x_{0}\right)+y^{* t}\left(x_{0}\right)$ for all $i=1,2, \ldots, k$, then $\lambda^{* t} f\left(u^{*}\right)+$ $y^{* t} g\left(u^{*}\right)>\lambda^{* t} f\left(x_{0}\right)+y^{* t} g\left(x_{0}\right)$. Since $\lambda^{* t} f+y^{* t} g$ is pseudoconvex, it follows that

$$
\left(x_{0}-u^{*}\right)^{t}\left\{\nabla \lambda^{* t} f\left(u^{*}\right)+\nabla y^{* t} g\left(u^{*}\right)\right\}<0
$$

contradicting the feasibility of $\left(u^{*}, y^{*}, \lambda^{*}\right)$.

Hence $\left(x_{0}, y, \lambda\right)$ is a weak maximum for $(D)$.

Remark 3.3. Theorems 3.1 and 3.2 give multiple objective extensions of the results of Bector et al [1] and Mahajan and Vartak [10] for scalar valued minimisation problems.

Mond and Weir [12] proposed a number of different duals to the scalar valued minimisation problem. Here it is shown, as for the Wolfe dual, that there are analogous results for the multiple objective optimisation problem $(P)$.

In relation to $(P)$ consider the problem

(D1) maximise $f(u)$

$$
\begin{aligned}
\text { subject to } \nabla \lambda^{t} f(u)+\nabla y^{t} g(u) & =0, \\
y^{t} g(u) & \geqq 0, \\
y & \geqq 0, \\
\lambda & \in \Lambda,
\end{aligned}
$$

where $\Lambda=\left\{\lambda \in \mathbf{R}^{k}: \lambda \geqslant 0, \lambda^{t} e=1\right\}$.

Theorem 3.4. (Weak Duality) If, for all feasible $(x, u, y, \lambda)$

(a) $f$ is pseudoconvex and $y^{t} g$ is quasiconvex; or

(b) $\lambda^{t} f$ is pseudoconvex and $y^{t} g$ is quasiconvex; or

(c) $f$ is quasiconvex and $y^{t} g$ is strictly pseudoconvex; or

(d) $\lambda^{t} f$ is quasiconvex and $y^{t} g$ is strictly pseudoconvex, then $f(x) \nless f(u)$.

ProOF:

(a) Let $x$ be feasible for $(P)$ and $(u, y, \lambda)$ feasible for $(D 1)$. Suppose $f_{i}(x)<f_{i}(u)$ for all $i=1,2, \ldots, k$. By pseudoconvexity of $f_{i}, i=1,2, \ldots, k$

$$
(x-u)^{t} \nabla f_{i}(u)<0, i=1,2, \ldots, k .
$$

Since $\lambda \geqslant 0$,

$$
(x-u)^{t} \nabla \lambda^{t} f(u)<0
$$


Since $y^{t} g(x)-y^{t} g(u) \leqq 0$, the quasiconvexity of $y^{t} g$ implies that

$$
(x-u)^{t} \nabla y^{t} g(u) \leqq 0 .
$$

Combining (15) and (16) gives

$$
(x-u)^{t}\left\{\nabla \lambda^{t} f(u)+\nabla y^{t} g(u)\right\}<0
$$

which contradicts the constraint (11) of $(D 1)$.

(b) Let $x$ be feasible for $(P)$ and $(u, y, \lambda)$ feasible for $(D 1)$. Suppose $f_{i}(x)<f_{i}(u)$ for all $i=1,2, \ldots, k$. Since $\lambda \geqslant 0$ it follows that

$$
\lambda^{t} f(x)<\lambda^{t} f(u)
$$

and pseudoconvexity of $\lambda^{t} f$ implies

$$
(x-u)^{t} \nabla \lambda^{t} f(u)<0 .
$$

Since $y^{t} g(x)-y^{t} g(u) \leqq 0$ the quasiconvexity of $y^{t} g$ implies that

$$
(x-u)^{t} \nabla y^{t} g(u) \leqq 0 .
$$

Combining (17) and (18) gives

$$
(x-u)^{t}\left\{\nabla \lambda^{t} f(u)+\nabla y^{t} g(u)\right\}<0
$$

which contradicts the constraint (11) of $(D 1)$.

(c) Let $x$ be feasible for $(P)$ and $(u, y, \lambda)$ feasible for $(D 1)$. Suppose $f_{i}(x)<f_{i}(u)$ for all $i=1,2, \ldots, k$. The quasiconvexity of $f_{i}, i=1,2, \ldots, k$ implies that

$$
(x-u)^{t} \nabla f_{i}(u) \leqq 0
$$

and since $\lambda \geqslant 0$

$$
(x-u)^{t} \nabla \lambda^{t} f(u) \leqq 0 .
$$

By (11)

$$
(x-u)^{t} \nabla y^{t} g(u) \geqq 0
$$

and since $y^{t} g$ is strictly pseudoconvex

$$
y^{t} g(x)>y^{t} g(u)
$$

which is a contradiction since $y^{t} g(x) \leqq 0$ and $y^{t} g(u) \geqq 0$. 
(d) Let $x$ be feasible for $(P)$ and $(u, y, \lambda)$ feasible for $(D 1)$. Suppose $f_{i}(x)<f_{i}(u)$ for all $i=1,2, \ldots, k$. Since $\lambda \geqslant 0, \lambda^{t} f(x)<\lambda^{t} f(u)$, the quasiconvexity of $\lambda^{t} f$ implies that

$$
(x-u)^{t} \nabla \lambda^{t} f(u) \leqq 0
$$

By (11)

$$
(x-u)^{t} \nabla y^{t} g(u) \geqq 0
$$

and since $y^{t} g$ is strictly pseudoconvex

$$
y^{t} g(x)>y^{t} g(u)
$$

which is a contradiction since $y^{t} g(x) \leqq 0$ and $y^{t} g(u) \geqq 0$.

Theorem 3.5. (Strong Duality) Let $x_{0}$ be a weak minimum for $(P)$ at which the Kuhn-Tucker constraint qualification is satisfied. Then there exist $(y, \lambda)$ such that $\left(x_{0}, y, \lambda\right)$ is feasible for $(D 1)$ and the objective values of $(P)$ and $(D 1)$ are equal. If, also,

(a) $f$ is pseudoconvex and $y^{t} g$ is quasiconvex; or

(b) $\lambda^{t} f$ is pseudoconvex and $y^{t} g$ is quasiconvex; or

(c) $f$ is quasiconvex and $y^{t} g$ is strictly pseudoconvex; or

(d) $\lambda^{t} f$ is quasiconvex and $y^{t} g$ is strictly pseudoconvex, then $\left(x_{0}, y, \lambda\right)$ is a weak maximum for $(D 1)$.

Proof: Since $x_{0}$ is a weak minimum for $(P)$ at which the Kuhn-Tucker constraint qualification is satisfied, then by Theorem 2.2 , there exists $y \geqq 0, \lambda \geqslant 0, \lambda^{t} e=1$ such that

$$
\nabla \lambda^{t} f\left(x_{0}\right)+\nabla y^{t} g\left(x_{0}\right)=0, y^{t} g\left(x_{0}\right)=0
$$

Thus $\left(x_{o}, y, \lambda\right)$ is feasible for $(D 1)$ and clearly the objective values of $(P)$ and (D1) are equal.

If $\left(x_{0}, y, \lambda\right)$ is not a weak maximum for $(D 1)$ there exists feasible $\left(u^{*}, y^{*}, \lambda^{*}\right)$ for (D1) such that $f_{i}\left(u^{*}\right)>f_{i}\left(x_{0}\right)$ for all $i=1,2, \ldots, k$.

(a) Since $f$ is pseudoconvex $\left(x_{0}-u^{*}\right)^{t} \nabla f_{i}\left(u^{*}\right)<0$ for all $i=1,2, \ldots, k$. Thus, since $\lambda^{*} \geqslant 0$,

$$
\left(x_{0}-u^{*}\right)^{t} \nabla \lambda^{* t} f\left(u^{*}\right)<0 .
$$

Also $y^{* t} g\left(x_{0}\right)-y^{* t} g\left(u^{*}\right) \leqq 0$ and since $y^{* t} g$ is quasiconvex

$$
\left(x_{0}-u^{*}\right)^{t} \nabla y^{* t} g\left(u^{*}\right) \leqq 0
$$


Combining (19) and (20) gives

$$
\left(x_{0}-u^{*}\right)^{t}\left\{\nabla \lambda^{* t} f\left(u^{*}\right)+\nabla y^{* t} g\left(u^{*}\right)\right\}<0
$$

which contradicts the feasibility of $\left(u^{*}, y^{*}, \lambda^{*}\right)$.

(b) Since $\lambda \geqslant 0$, then

$$
\lambda^{* t} f\left(u^{*}\right)>\lambda^{* t} f\left(x_{0}\right)
$$

Since $\lambda^{* t} f$ is pseudoconvex then

$$
\left(x_{0}-u^{*}\right)^{t} \nabla \lambda^{* t} f\left(u^{*}\right)<0
$$

Also $y^{* t} g\left(x_{0}\right)-y^{* t} g\left(u^{*}\right) \leqq 0$ and since $y^{* t} g$ is quasiconvex

$$
\left(x_{0}-u^{*}\right)^{t} \nabla y^{* t} g\left(u^{*}\right) \leqq 0
$$

Combining (21) and (22) gives

$$
\left(x_{0}-u^{*}\right)^{t}\left\{\nabla \lambda^{* t} f\left(u^{*}\right)+\nabla y^{* t} g\left(u^{*}\right)\right\}<0
$$

which contradicts the feasibility of $\left(u^{*}, y^{*}, \lambda^{*}\right)$.

(c) Since $f$ is quasiconvex $\left(x_{0}-u^{*}\right)^{t} \nabla f_{i}\left(u^{*}\right) \leqq 0$ for all $i=1,2, \ldots, k$. Since $\lambda \geqslant 0$

$$
\left(x_{0}-u^{*}\right)^{t} \nabla \lambda^{* t} f\left(u^{*}\right) \leqq 0 .
$$

By (11)

$$
\left(x_{0}-u^{*}\right)^{t} \nabla y^{* t} g\left(u^{*}\right) \geqq 0
$$

and since $y^{m t} g$ is strictly pseudoconvex

$$
y^{* t} g\left(x_{0}\right)>y^{* t} g\left(u^{*}\right)
$$

This is a contradiction as $y^{* t} g\left(x_{0}\right) \leqq 0$ and $y^{* t} g\left(u^{*}\right) \geqq 0$.

(d) Since $\lambda^{*} \geqslant 0, \lambda^{* t} f\left(u^{*}\right)>\lambda^{* t} f\left(x_{0}\right)$. Thus, since $\lambda^{* t} f$ is quasiconvex

$$
\left(x_{0}-u^{*}\right)^{t} \nabla \lambda^{* t} f\left(u^{*}\right) \leqq 0 .
$$

By (11)

$$
\left(x_{0}-u^{*}\right)^{t} \nabla y^{* t} g\left(u^{*}\right) \geqq 0
$$

and since $y^{* t} g$ is strictly pseudoconvex

$$
y^{* t} g\left(x_{0}\right)>y^{* t} g\left(u^{*}\right)
$$


This is a contradiction as $y^{* t} g\left(x_{0}\right) \leqq 0$ and $y^{* t} g\left(u^{*}\right) \geqq 0$.

In a similar manner to that given in [12] we state a general dual for the multiple objective optimisation problem. For completeness we shall consider the case where the primal problem has equality as well as inequality constraints.

Consider the problem:

(PE) minimise $f(x)$

$$
\begin{aligned}
\text { subject to } g(x) & \leqq 0 \\
h(x) & =0
\end{aligned}
$$

where $f: \mathbf{R}^{n} \rightarrow \mathbf{R}^{k}, g: \mathbf{R}^{n} \rightarrow \mathbf{R}^{m}, h: \mathbf{R}^{n} \rightarrow \mathbf{R}^{\ell}$ are all differentiable.

Let $M=\{1,2, \ldots, m\}, L=\{1,2, \ldots, l\}, I_{\alpha} \subseteq M, \alpha=0,1,2, \ldots, \nu$ with $I_{\alpha} \cap I_{\beta}=$ $\phi, \alpha \neq \beta$ and $\bigcup_{\alpha=0}^{\nu} I_{\alpha}=M$ and $J_{\alpha} \subseteq L, \alpha=0,1,2, \ldots, \nu$, with $J_{\alpha} \cap J_{\beta}=\phi, \alpha \neq \beta$ and $\bigcup_{\alpha=0}^{\nu} J_{\alpha}=L$

Note that any particular $I_{\alpha}$ or $J_{\alpha}$ may be empty. Thus if $M$ has $\nu_{1}$ disjoint subsets and $L$ has $\nu_{2}$ disjoint subsets, $\nu=\operatorname{Max}\left[\nu_{1}, \nu_{2}\right]$. So that if $\nu_{1}>\nu_{2}$, then $J_{\alpha}, \alpha>\nu_{2}$ is empty.

In relation to $(P E)$ consider the problem:

(DE) maximise $f(u)+\sum_{i \in I_{0}} y_{i} g_{i}(u) e+\sum_{j \in J_{0}} z_{j} h_{j}(u) e$

$$
\begin{aligned}
& \text { subject to } \nabla \lambda^{t} f(u)+\nabla y^{t} g(u)+\nabla z^{t} h(u)=0, \\
& \qquad \sum_{i \in I_{\alpha}} y_{i} g_{i}(u)+\sum_{j \in J_{\alpha}} z_{j} h_{j}(u) \\
& \geqq 0, \alpha=1,2, \ldots, \nu, \\
& y \geqq 0, \\
& \lambda \in \Lambda,
\end{aligned}
$$

where $\Lambda=\left\{\lambda \in \mathbf{R}^{k}: \lambda \geqslant 0, \lambda^{t} e=1\right\}$.

The following weak and strong duality theorems are stated without proof. They may be established in a manner similar to Theorems 3.1, 3.2, 3.4 and 3.5.

Theorem 3.6. (Weak Duality) If, for all feasible $(x, u, y, z, \lambda)$

(a) $f+\sum_{i \in I_{0}} y_{i} g_{i} e+\sum_{j \in J_{0}} z_{j} h_{j} e$ is pseudoconvex and $\sum_{i \in I_{\alpha}} y_{i} g_{i}+\sum_{j \in J_{\alpha}} z_{j} h_{j}, \alpha=$ $1,2, \ldots, \nu$ is quasiconvex; or 
(b) $\lambda^{t} f+\sum_{i \in I_{0}} y_{i} g_{i}+\sum_{j \in J_{0}} z_{j} h_{j}$ is pseudoconvex and $\sum_{i \in I \alpha} y_{i} g_{i}+\sum_{j \in J \alpha} z_{j} h_{j}, \alpha=$ $1,2, \ldots, \nu$ is quasiconvex; or

(c) $I_{0} \neq M$ and $J_{0} \neq L, f+\sum_{i \in I_{0}} y_{i} g_{i} e+\sum_{j \in J_{0}} z_{j} h_{j} e$ is quasiconvex and $\sum_{i \in I_{\alpha}} y_{i} g_{i}+\sum_{j \in J_{\alpha}} z_{j} h_{j}, \alpha=1,2, \ldots, \nu$ is strictly pseudoconvex; or

(d) $I_{0} \neq M$ and $J_{0} \neq L, \lambda^{t} f+\sum_{i \in I_{0}} y_{i} g_{i}+\sum_{j \in J_{0}} z_{j} h_{j}$ is quasiconvex and $\sum_{i \in I_{\alpha}} y_{i} g_{i}+\sum_{j \in J_{\alpha}} z_{j} h_{j}, \alpha=1,2, \ldots, \nu$ is strictly pseudoconvex, then

$$
f(x) \nless f(u)+\sum_{i \in I_{0}} y_{i} g_{i}(u) e+\sum_{j \in J_{0}} z_{j} h_{j}(u) e .
$$

Theorem 3.7. (Strong Duality) Let $x_{0}$ be a weak minimum for $(P E)$ at which the Kuhn-Tucker constraint qualification is satisfied. Then there exist $(y, z, \lambda)$ such that $\left(x_{0}, y, z, \lambda\right)$ is feasible for $(D E)$ and the objective values of $(P E)$ and $(D E)$ are equal. If also the assumptions (a), (b), (c) or (d) of Theorem 3.6 are satisfied; then $\left(x_{0}, y, z, \lambda\right)$ is a weak maximum for $(D E)$.

\section{Converse Duality}

THEOREM 4.1. Let $\left(x_{0}, y_{0}, \lambda_{0}\right)$ be a weak maximum of $(D 1)$. Assume the $n \times n$ Hessian matrix

$$
\nabla^{2} \lambda_{0}^{t} f\left(x_{0}\right)+\nabla^{2} y_{0}^{t} g\left(x_{0}\right)
$$

is positive or negative definite and the vectors $\nabla f_{i}\left(x_{0}\right), i=1,2, \ldots, k$ are linearly independent. If for all feasible $(x, u, y, \lambda)$

(a) $f$ is pseudoconvex and $y^{t} g$ is quasiconvex; or

(b) $\lambda^{t} f$ is pseudoconvex and $y^{t} g$ is quasiconvex; or

(c) $f$ is quasiconvex and $y^{t} g$ is strictly pseudoconvex; or

(d) $\lambda^{t} f$ is quasiconvex and $y^{t} g$ is strictly pseudoconvex, then $x_{0}$ is a weak minimum for $(P)$.

Proof: Since $\left(x_{0}, y_{0}, \lambda_{0}\right)$ is a weak maximum for $(D)$, then by Theorem 2.1 there exist

$$
\tau \in \mathbf{R}^{k}, \nu \in \mathbf{R}^{n}, p \in \mathbf{R}, s \in \mathbf{R}^{m}, w \in \mathbf{R}^{k}
$$

such that 


$$
\begin{aligned}
\nabla \tau^{t} f\left(x_{0}\right)+\nabla \nu^{t}\left[\nabla \lambda_{0}^{t} f\left(x_{0}\right)+\nabla y_{0}^{t} g\left(x_{0}\right)\right]+p \nabla y_{0}^{t} g\left(x_{0}\right) & =0, \\
\left(\nabla g\left(x_{0}\right)\right)^{t} \nu+p g\left(x_{0}\right)+s & =0, \\
\left(\nabla f\left(x_{0}\right)\right)^{t} \nu+w & =0, \\
p y_{0}^{t} g\left(x_{0}\right) & =0, \\
s^{t} y_{0} & =0, \\
w^{t} \lambda_{0} & =0, \\
(\tau, s, p, w) & \geqq 0, \\
(\tau, \nu, s, p, w) & \neq 0 .
\end{aligned}
$$

Since $\lambda_{0} \in \Lambda,(29)$ gives $w=0 ;(26)$ then gives

$$
\nu^{t} \nabla f\left(x_{0}\right)=0 .
$$

Multiplying (25) by $y_{0}$ and using (27) and (28) gives

$$
\nu^{\ell} \nabla y_{0}^{t} g\left(x_{0}\right)=0 .
$$

Multiplying (24) by $\nu^{t}$ and using (32) and (33) gives

$$
\nu^{t}\left[\nabla^{2} \lambda_{0}^{t} f\left(x_{0}\right)+\nabla^{2} y_{0}^{t} g\left(x_{0}\right)\right] \nu=0 .
$$

Since (23) is assumed positive or negative definite, $\nu=0$.

Since $\nu=0,(24)$ and the equality constraint (11) of $(D 1)$ give

$$
\nabla\left(\tau-p \lambda_{0}\right)^{t} f\left(x_{0}\right)=0 .
$$

By the linear independence of $\nabla f_{i}\left(x_{0}\right), i=1,2, \ldots, k$ it follows that

$$
\tau=p \lambda_{0}
$$

Since $\lambda_{0} \geqslant 0, \tau=0$ implies $p=0$ and then, by (25), $s=0$ giving $(\tau, \nu, s, p, w)=0$ contradicting (31). Thus $\tau \neq 0$ and $p>0$. Since $\nu=0, p>0$ and $s \geqq 0,(25)$ gives $g\left(x_{0}\right) \leqq 0$, and (27) gives $y_{0}^{t} g\left(x_{0}\right)=0$. Thus $x_{0}$ is feasible for $(P)$. That $x_{0}$ is a weak minimum for $(P)$ then follows under assumptions (a), (b), (c) or (d) from weak duality, Theorem 3.4.

As in [12] a more general converse duality result may be established for $(P E)$ and $(D E)$. The proof follows in a manner similar to that of Theorem 4.1. 
Theorem 4.2. Let $\left(x_{0}, y_{0}, z_{0}, \lambda_{0}\right)$ be a weak maximum of $(D E)$. Assume the $n \times n$ Hessian matrix

$$
\nabla^{2} \lambda_{0}^{t} f\left(x_{0}\right)+\nabla^{2} y_{0}^{t} g\left(x_{0}\right)+\nabla^{2} z_{0}^{t} h\left(x_{0}\right)
$$

is positive or negative definite and that the set

$$
\left\{\nabla f_{i}\left(x_{0}\right), i=1,2, \ldots, k, \sum_{i \in I_{\alpha}} \nabla y_{0 i} g_{i}\left(x_{0}\right)+\sum_{j \in J_{\alpha}} \nabla z_{0 j} h_{j}\left(x_{0}\right), \alpha=1,2, \ldots, \nu\right\}
$$

is linearly independent whenever $I_{0} \neq M$ or $J_{0} \neq L$.

If the assumptions (a), (b), (c) or (d) of Theorem 3.6 hold, then $x_{0}$ is a weak minimum for $(P E)$.

In the case $I_{0}=M$ and $L=\phi$ this result simplifies slightly.

Theorem 4.3. Let $\left(x_{0}, y_{0}, \lambda_{0}\right)$ be a weak maximum of $(D)$. Assume the $n \times n$ Hessian matrix

$$
\nabla^{2} \lambda_{0}^{t} f\left(x_{0}\right)+\nabla^{2} y_{0}^{t} g\left(x_{0}\right)
$$

is positive or negative definite. If the assumptions (a) or (b) of Theorem 3.1 hold, then $x_{0}$ is a weak minimum of $(P)$.

Theorems 4.1, 4.2 and 4.3 give multiple objective generalisations of the scalar valued programming results of Mond and Weir [12], Bector and Bector [2] and Craven and Mond [5].

We now turn our attention to strict converse duality.

Theorem 4.4. Let $x_{0}$ be a weak minimum for $(P)$ and $\left(u_{0}, y_{0}, \lambda_{0}\right)$ be a weak maximum for $(D 1)$ such that $\lambda_{0}^{t} f\left(x_{0}\right) \leqq \lambda_{0}^{t} f\left(u_{0}\right)$. Assume that

(a) $\lambda_{0}^{t} f$ is strictly pseudoconvex at $u_{0}$ and $y_{0}^{t} g$ is quasiconvex at $u_{0}$; or

(b) $\lambda_{0}^{t} f$ is quasiconvex at $u_{0}$ and $y_{0}^{t} g$ is strictly pseudoconvex at $u_{0}$; then $x_{0}=u_{0}$; that is, $u_{0}$ is a weak minimum for $(P)$.

Proof:

(a) We assume $x_{0} \neq u_{0}$ and exhibit a contradiction. Since $x_{0}$ and $\left(u_{0}, y_{0}, \lambda_{0}\right)$ are feasible for $(P)$ and $(D 1)$ respectively

$$
y_{0}^{t} g\left(x_{0}\right)-y_{0}^{t} g\left(u_{0}\right) \leqq 0
$$

and the quasiconvexity of $y_{0}^{t} g$ at $u_{0}$ implies

$$
\left(x_{0}-u_{0}\right)^{t} \nabla y_{0}^{t} g\left(u_{0}\right) \leqq 0 .
$$


From (11), $\left(x_{0}-u_{0}\right)^{t} \nabla \lambda_{0}^{t} f\left(u_{0}\right) \geqq 0$ and, by the strict pseudoconvexity of $\lambda_{0}^{t} f$ at $u_{0}$,

$$
\lambda_{0}^{t} f\left(x_{0}\right)>\lambda_{0}^{t} f\left(u_{0}\right)
$$

contradicting the assumption that $\lambda_{0}^{t} f\left(x_{0}\right) \leqq \lambda_{0}^{t} f\left(u_{0}\right)$.

(b) We assume $x_{0} \neq u_{0}$ and exhibit a contradiction. Since $x_{0}$ and $\left(u_{0}, y_{0}, \lambda_{0}\right)$ are feasible for $(P)$ and $(D 1)$ respectively

$$
y_{0}^{t} g\left(x_{0}\right)-y_{0}^{t} g\left(u_{0}\right) \leqq 0
$$

and the strict pseudoconvexity of $y_{0}^{t} g$ at $u_{0}$ implies

$$
\left(x_{0}-u_{0}\right)^{t} \nabla y_{0}^{t} g\left(u_{0}\right)<0
$$

From (11), $\left(x_{0}-u_{0}\right)^{t} \nabla \lambda_{0}^{t} f\left(u_{0}\right)>0$ and, by quasiconvexity of $\lambda_{0}^{t} f$ at $u_{0}$,

$$
\lambda_{0}^{t} f\left(x_{0}\right)>\lambda_{0}^{t} f\left(u_{0}\right)
$$

contradicting the assumption that $\lambda_{0}^{t} f\left(x_{0}\right) \leqq \lambda_{0}^{t} f\left(u_{0}\right)$.

THEOREM 4.5. Let $x_{0}$ be a weak minimum for $(P E)$ and $\left(u_{0}, y_{0}, z_{0}, \lambda_{0}\right)$ be a weak maximum for $(D E)$ such that $\lambda_{0}^{t} f\left(x_{0}\right) \leqq \lambda_{0}^{t} f\left(u_{0}\right)+\sum_{i \in I_{0}} y_{0 i} g_{i}\left(u_{0}\right)+\sum_{j \in J_{0}} z_{0 j} h_{j}\left(u_{0}\right)$. If

(a) $\lambda_{0}^{t} f+\sum_{i \in I_{0}} y_{0 i} g_{i}+\sum_{j \in J_{0}} z_{0 j} h_{j}$ is strictly pseudoconvex at $u_{0}$ and each $\sum_{i \in I_{\alpha}} y_{0 i} g_{i}+\sum_{j \in J_{\alpha}} z_{0 j} h_{j}, \alpha=1,2, \ldots, \nu$ is quasiconvex at $u_{0}$; or

(b) $\lambda_{0}^{t} f+\sum_{i \in I_{0}} y_{0 i} g_{i}+\sum_{j \in J_{0}} z_{0 j} h_{j}$ is quasiconvex at $u_{0}$ and each

$$
\sum_{i \in I_{\alpha}} y_{0 i} g_{i}+\sum_{j \in J_{\alpha}} z_{0 j} h_{j}, \alpha=1,2, \ldots, \nu
$$

is strictly pseudoconvex at $u_{0}$,

then $x_{0}=u_{0}$; that is $u_{0}$ is a weak minimum for $(P)$.

COROLlary 4.6. Let $x_{0}$ be a weak minimum for $(P)$ and $\left(u_{0}, y_{0}, \lambda_{0}\right)$ be a weak maximum for $(D)$ such that $\lambda_{0}^{t} f\left(x_{0}\right) \leqq \lambda_{0}^{t} f\left(u_{0}\right)+y_{0}^{t} g\left(u_{0}\right)$. If $\lambda_{0}^{t} f+y_{0}^{t} g$ is strictly pseudoconvex at $u_{0}$, then $x_{0}=u_{0}$; that is $u_{0}$ is a weak minimum for $(P)$.

These strict converse duality results give multiple objective analogues of the scalar programming theorems of Mond and Weir [12], Bector and Bector [2], Weir [14, 15] and Mahajan and Vartak [10]. 


\section{REFERENCES}

[1] C.R. Bector M.K. Bector and J.E. Klassen, 'Duality for a nonlinear programming problem', Utilitas Math 11 (1977), 87-99.

[2] C.R. Bector and M.K. Bector, 'On various duality theorems in nonlinear progranming', J. Optim. Theory Appl 53 (1987), 509- 515.

[3] G. Bitran, 'Duality in nonlinear multiple criteria optimization problems', J. Optim. Theory Appl. 35 (1981), 367-406.

[4] B.D. Corley, 'Duality theory for maximizations with respect to cones', J. Math. Anal. Appl 84 (1981), 560-568.

[5] B.D. Craven and B. Mond, 'On converse duality in nonlinear programming', Oper. Res 19 (1971), 1075-1078.

[6] B.D. Craven, 'Langrangian conditions and quasiduality', Bull. Austral. Math. Soc 16 (1977), 325-339.

[7] B.D. Craven, 'Strong vector minimization and duality', $Z$. Angew. Math. Mech 60 (1980), 1-5.

[8] A.M. Geoffrion, 'Proper efficiency and the theory of vector maximization', J. Math. Anal. Appl $22(1968), 618-630$.

[9] E. H. Ivanov and R. Nehse, 'Some results on dual vector optimization problems', Optimization 16 (1985), 505-517.

[10] D. G. Mahajan and M.N. Vartak, 'Generalization of some duality theorems in nonlinear programming', Math. Programming 12 (1977), 293-317.

[11] O. L. Mangasarian, Nonlinear programming (McGraw-Hill, New York, 1969).

[12] B. Mond and T. Weir, 'Generalized concavity and duality', in Generalized concavity in optimiza. tion and economics, Edited by S. Schaible and W.T. Ziemba, pp. 263-279 (Academic Press, New York, 1981).

[13] T. Tanino and Y. Sawaragi, 'Duality theory in multiobjective programming', J. Optim. Theory Appl 27 (1979), 509-529.

[14] T. Weir, 'Generalized Convexity and Duality in Mathematical Programming', Ph.D. Thesis. (La Trobe University, Bundoora, Melbourne, Australia, 1982).

[15] T. Weir, 'A note on strict converse duality in nonlinear programming', J. Inf. Opt. Sci 7 (1986), 65-71.

[16] T. Weir, 'Proper efficiency and duality for vector valued optimization problems', J. Austral. Math. Soc., Series A 43 (1987), 21-34.

[17] D. G. White, 'Vector maximization and Lagrange multipliers', Math. Programming 31 (1985), 192-205.

[18] P. Wolfe, 'A duality theorem for nonlinear programming', Quart. Appl. Math 19 (1961), 239-244.

Dr T. Weir

7/35 Gaza Rd.,

West Ryde NSW 2114

Australia
Professor B. Mond

Department of Mathematics

La Trobe University

Bundoora, Victoria, 3083

Australia 